\title{
A review of Zika virus infections in pregnancy and implications for antenatal care in Singapore
}

\author{
Harvard Zhenjia Lin ${ }^{1}$, MBBS, MRCoG, Paul Anantharajah $\underline{\text { Tambyah }}^{2}$, MD, FRCP, Eu Leong Yong ${ }^{1}$, FRCOG, PhD, \\ Arijit Biswas $^{1}$, MD, FRCOG, Shiao-Yng $\underline{\text { Chan }}^{1}$, FRCOG, PhD
}

\begin{abstract}
Given the consensus that there is a causal relationship between Zika virus (ZIKV) infection in pregnancy and congenital Zika syndrome (CZS), clinicians must be prepared to manage affected patients despite the numerous gaps in current knowledge. The clinical course in pregnancy appears similar to that in non-pregnant women, although viraemia may be prolonged. ZIKV infection can be diagnosed by serum and urine reverse transcription-polymerase chain reaction, but commercially available serological tests are currently unreliable in dengue-endemic regions. Although vertical transmission can occur at any time during gestation, first- and second-trimester infections have the highest risk of developing central nervous system anomalies. Aberrant fetal growth and pregnancy loss may also occur. Serial ultrasonography should be conducted for infected cases. Without a vaccine, pregnant women should be advised to minimise mosquito bites and reduce sexual transmission risk. Overall, the absolute risk of CZS arising amid a ZIKV outbreak appears relatively low.
\end{abstract}

Keywords: infectious disease transmission (vertical), fetal development, nervous system malformations, pregnancy, Zika virus

\section{INTRODUCTION}

In February 2016, the World Health Organization (WHO) declared the Zika virus (ZIKV) outbreak in South America and the associated increase in neurological disorders and neonatal malformations a "public health emergency of international concern". Subsequently, in April 2016, the United States Centers for Disease Control and Prevention (CDC) and WHO concluded that there was sufficient evidence to support a causal relationship between maternal ZIKV infection and congenital Zika syndrome (CZS) in offspring. ${ }^{(1)}$ From the end of August 2016, Singapore saw a rapid rise in the number of ZIKV infections, with over 200 cases within a week of the first locally acquired infection, including two pregnant cases. This review seeks to summarise the clinical features of ZIKV infection in pregnancy from case reports and case series, and to discuss existing guidelines, in order to help clinicians working in regions with substantial Aedes mosquito populations to perform patient counselling and decision-making despite the numerous gaps in current knowledge.

\section{A BRIEF HISTORY OF THE ZIKA VIRUS}

ZIKV is a single-stranded RNA, Flavivirus, which was first isolated from a febrile sentinel Rhesus monkey in Uganda in 1947..$^{(2,3)}$ The Flaviviridae family includes the Dengue virus, West Nile virus, tick-borne encephalitis virus and yellow fever virus. As with dengue fever, the Aedes mosquito is the primary route of ZIKV transmission between humans, and vector control measures remain the primary line of defence in the absence of a proven vaccine.

Reported cases of ZIKV infections in previous decades were not confined to Africa, but also included cases from Asia, resulting from African and Asian strains of ZIKV, respectively. In particular, evidence for the long-standing presence of ZIKV in Southeast
Asia comes from indirect serological evidence in non-acute samples from Thailand, ${ }^{(4)}$ Malaysia, ${ }^{(5)}$ Indonesia ${ }^{(6)}$ and Vietnam ${ }^{(7)}$ between the 1950s and 1970s. Through the years, sporadic cases have been documented across Africa, Australia/Oceania, South America and Asia. ${ }^{(8)}$ The recent surge in the number of cases of active local transmission of the Southeast Asian ZIKV strain in Singapore suggests an increase in the occurrence of factors such as El Niño weather conditions, ${ }^{(9)}$ which can promote transmission of locally present ZIKV. It is also highly likely that a subset of the Singapore population may be immune to ZIKV. However, there have been no reports in recent years determining the specific seroprevalence rates of ZIKV in the region.

Over the past decade, several outbreaks of the Asian strain of ZIKV have been described in the Yap Island of Micronesia (2007), ${ }^{(10)}$ French Polynesia (2013-2014) ${ }^{(11)}$ and the Pacific Islands (2014-2016), ${ }^{(12)}$ none of which initially described associations with pregnancy complications at the time, although a sharp rise in cases of Guillain-Barré syndrome (GBS) was noted in French Polynesia. The current epidemic in Brazil was thought to have originated in Easter Island, Chile, either during the cultural games (which the French Polynesians attended) or from an international canoe competition. ${ }^{(13)}$ ZIKV spread very quickly to Brazil. Since the first detection of ZIKV in Brazil in March 2015, ${ }^{(14)}$ the virus has spread throughout the Americas. Up till 19 May 2016, 60 countries and territories have reported cases of ZIKV infections. ${ }^{(15)}$

The Brazilian outbreak coincided with an unexpected upsurge in the incidence of microcephaly, central nervous system (CNS) malformations and neurological disorders, including GBS in adults. ${ }^{(16-18)}$ Subsequently, French Polynesia also reported an increase in microcephaly cases during the ZIKV outbreak of 2014-2015. ${ }^{(19)}$ Although a 20-fold rise in the incidence of microcephaly in Brazil has been reported, ${ }^{(20)}$ this figure was

${ }_{1}^{1}$ Department of Obstetrics and Gynaecology, ${ }^{2}$ Department of Medicine (Infectious Disease), Yong Loo Lin School of Medicine, National University of Singapore, Singapore Correspondence: A/Prof Chan Shiao-Yng, Associate Professor and Consultant, Department of Obstetrics and Gynaecology, Yong Loo Lin School of Medicine, National University of Singapore, 1E Kent Ridge Road, NUHS Tower Block, Level 12, Singapore 119228. obgchan@nus.edu.sg 
marred by controversy due to an unclear baseline incidence rate, inconsistent case definitions and variable reporting practices.

Given the potentially serious implications of ZIKV infection in pregnancy, the importance of such research has now been prioritised, but to date there are a limited number of studies published on this area. Using the key search terms 'Zika' and 'pregnant', we identified and reviewed published articles through PubMed, as well as the WHO, American College of Obstetricians and Gynecologists (ACOG), CDC, European Centre for Disease Prevention and Control and Singapore's Ministry of Health $(\mathrm{MOH})$ websites. We identified over 20 primary articles describing cases of ZIKV infection in pregnancy comprising either the mother or fetus/neonate, and have summarised them in this review. We considered the two largest cohorts: an ongoing Colombian public health surveillance system that includes the largest documented prospective cohort of pregnant women $(n=1,850)$, all of whom had confirmed symptomatic ZIKV infection; ${ }^{(21)}$ and a retrospective case series comprising 602 neonatal cases of definite and probable CZS. ${ }^{(22)}$

\section{IS THE CLINICAL COURSE OF ZIKV INFECTION DIFFERENT IN PREGNANCY?}

The incubation period after being bitten by the Aedes mosquito is estimated to be 3-12 days, ${ }^{(23)}$ with a median of about six days. ${ }^{(24)}$ The only published evidence in pregnancy comes from a case report of a pregnant woman and her husband who had both travelled to Guatemala and developed symptoms five days after their return, ${ }^{(25)}$ suggesting that the incubation period is similar during pregnancy. The Yap survey of infected residents, identified through positivity for the ZIKV immunoglobulin M (IgM) antibody, reported that only $19 \%$ of participants had a clinical illness that was probably attributable to ZIKV infection. ${ }^{(10)}$ Whether a similarly low proportion develops symptoms during pregnancy has not been systematically determined.

The range of symptoms and signs in pregnancy is similar to that in non-pregnant adults, ${ }^{(10,26,27)}$ and is equally mild, nonspecific and self-limiting, ${ }^{(28-31)}$ lasting for a similar duration of 4-6 days. ${ }^{(18)}$ Of note, fever is not a predominant feature of ZIKV infection in symptomatic pregnancies, being present in only $28 \%-46 \%$; $^{(28-30)}$ even then, most fevers are very low grade $\left(<38^{\circ} \mathrm{C}\right)$. A maculopapular rash (sometimes pruritic) was the most common symptom affecting $44 \%-93 \%$ of cases, followed by conjunctivitis $(36 \%-58 \%)$, myalgia/arthralgia $(39 \%-64 \%)$, headache $(53 \%-62 \%)$ and lymphadenopathy (about 40\%). ${ }^{(18,21,28,30)}$ During pregnancy, there does not appear to be an increased risk of developing the rarer complications of myelitis and $\mathrm{GBS},{ }^{(32,33)}$ with only one single case of GBS reported in pregnancy so far. ${ }^{(34)}$

\section{DIAGNOSIS OF ZIKV INFECTION DURING PREGNANCY}

In non-pregnant subjects, the level of viraemia among ZIKVinfected patients is generally low ${ }^{(35)}$ and the viraemia resolves quickly, mostly within 3-7 days, although it has been reported to last up to ten days in isolated cases. ${ }^{(23)} \mathrm{MOH}$ (Singapore) and
CDC recommendations for diagnosis are via real-time reverse transcription-polymerase chain reaction (RT-PCR) of ZIKV RNA in serum within the first week of symptom onset and in urine within 14 days. ${ }^{(36,37)}$ Although urine RT-PCR is predicted to be more consistently positive up to 14 days of symptom onset, it could still be positive for up to 20 days in a sizeable proportion of cases. ${ }^{(38)}$ The longest time that a urine RT-PCR test has been reported as positive in pregnancy was 25 days after symptom onset. ${ }^{(39,40)}$ Viraemia in pregnant women can also be prolonged beyond the estimated time frame, with simultaneously negative urine RT-PCR, ${ }^{(39)}$ which suggests a greater persistence of ZIKV in the circulation. The longest reported case of viraemia was up to 70 days, ending only when the pregnancy was terminated; (25) this suggests that continued propagation could occur within the fetoplacental unit, re-infecting the mother, or that the ability of the maternal immune system to clear the virus may be impaired during pregnancy. The findings support the first hypothesis: ZIKV was detectable by RT-PCR in amniotic fluid up to 18 weeks after maternal infection, while simultaneously collected maternal serum and urine were negative, ${ }^{(41)}$ and viral replication occurred in neural cell cultures in vitro following inoculation with fetal brain extracts from an infected case. ${ }^{(25)}$ However, from the limited number of reported cases, prolonged viraemia does not appear to correlate with poor outcomes for the fetus or the pregnancy. ${ }^{(39)}$

In pregnancy, there also appears to be significant variation in the rates of viral clearance from different fluids. In a prospective case series, among 72 ZIKV-confirmed symptomatic women tested by RT-PCR within five days of symptom onset, only $47 \%$ of cases were positive for both serum and urine tests, while $83 \%$ were positive for serum only and $64 \%$ for urine only, ${ }_{,}^{(18)}$ which suggests that serum testing has greater sensitivity than urine testing. The advantage of testing for ZIKV in urine is due to its prolonged presence, lasting for as long as a month after symptom onset in pregnancy. ${ }^{(38-40)}$ Thus, the testing of more than one type of body fluid could improve diagnostic sensitivity in pregnancy.

With reports of locally acquired ZIKV infection, the Singapore $\mathrm{MOH}$ has recommended the investigation of pregnant women who develop symptoms suggestive of ZIKV infection. The current case definition for investigation includes fever, maculopapular rash and any of the following: arthralgia; myalgia; headache; and non-purulent conjunctivitis. Since fever is typically mild, testing should still be offered when there is clinical suspicion of ZIKV infection, even in cases not meeting the strict case definition. If testing is carried out within seven days of symptom onset, both urine and serum ZIKV RT-PCR should be performed, but if it is within 7-14 days of symptom onset, only urine RT-PCR is recommended. In the presence of a positive result in either specimen, the pregnant woman should be counselled and managed as a confirmed case of ZIKV infection. ${ }^{(37)}$ If these tests are delayed and conducted after the period of viraemia, a negative result may provide false reassurance.

At the time of consultation, if the symptoms had occurred more than two weeks ago, ZIKV IgM testing is currently not a recommended option locally, since commercially available serological tests for ZIKV in dengue-endemic regions are unreliable 
and, therefore, not advised. Zika-specific immunoglobulins are reported to have significant cross-reactivity with antibodies to other flaviviruses, resulting in an unacceptably high false-positive rate. ${ }^{(35)}$ Although the majority of ZIKV-infected pregnant women are asymptomatic, the $\mathrm{MOH}$ currently does not advocate routine screening for asymptomatic ZIKV infection in pregnancy, in contrast to the CDC guidelines, which recommend screening of exposed but asymptomatic women (living in non-dengue endemic regions), albeit with warnings of its limitations. ${ }^{(35)}$ However, the $\mathrm{MOH}$ recommends serum and urine RT-PCR screening of asymptomatic pregnant women whose partners have a confirmed ZIKV infection.

\section{CZS AND RISK OF VERTICAL TRANSMISSION}

CZS comprises a variety of features, including a range of CNS abnormalities (of which microcephaly is the most commonly observed), ophthalmic anomalies, fetal growth restriction and placental malfunction phenotypes, and perinatal demise. ${ }^{(19)}$ With follow-up of infants infected with ZIKV in utero, associations with musculoskeletal, hepatic, genitourinary and cardiac defects have since emerged..$^{(42)}$

Strong arguments have already been made for a causal link between maternal ZIKV infection and CZS, ${ }^{(43)}$ with the implication of a direct transplacental maternal-fetal transmission of ZIKV. Conclusions of causality were drawn in April 2016 using Shepard's criteria for proof of teratogenicity (where $4 / 6$ relevant criteria were met and $1 / 6$ partially met) and Bradford Hill's criteria for evidence of causation (where $7 / 8$ relevant criteria were met); the one criterion unmet in both lists was the lack of an animal model of CZS, which has since been fulfilled. ${ }^{(44)}$

There have been debates about why this association had not previously been noticed in Africa and Asia; why there has not been a similar dramatic increase in CZS in Brazil's neighbouring countries, which are also experiencing ZIKV outbreaks; ${ }^{(21)}$ and why $90 \%$ of CZS cases in Brazil are clustered in the Northeast Region. Suggested explanations include the possibility that the risk of CZS may be modulated by other local associated factors, such as specific pesticide exposure, ${ }^{(45)}$ co-infection with other viruses and socioeconomic factors. ${ }^{(46)}$ The relatively higher prevalence of maternal hyperglycaemia in Brazil has been suggested as an exacerbating risk factor, ${ }^{(47)}$ but this is unlikely to be a significant factor, given that the prevalence of diabetes mellitus and gestational diabetes mellitus in South Asia and Southeast Asia is even higher than in South America, yet no cases of CZS have been documented. Despite the very high phylogenetic concordance of the ZIKV isolated from infected fetal brains from the recent South American outbreak with the ZIKV obtained from the French Polynesian outbreak (greater than $99.6 \%$ concordance) and with the Asian strain (98\% concordance), some have suggested that a substantially more virulent virus may have emerged from recent mutations. ${ }^{(30)}$ If this is true, then the currently transmitted Southeast Asian ZIKV strain in Singapore may not result in the same increased risk of CZS as in Brazil. Furthermore, the herd immunity already present in African and Asian countries, where large proportions of the population may have been infected as children, may be protective against widespread CZS, and hence the significance of seemingly isolated CZS cases may not have been recognised. The sheer scale and speed of the ZIKV outbreak in the highly populous urban areas of Zika-naïve Brazil may simply have been what was needed for a congenital disorder with a relatively low incidence to become apparent.

In determining the magnitude of the risk of vertical transmission, there are two separate considerations: the rate of infection of the fetoplacental unit following maternal ZIKV infection; and should fetoplacental infection occur, the rate of clinically significant fetal sequelae (in this case, in the form of CZS). For ZIKV infection, both of these considerations are not fully understood. The challenges of estimating vertical transmission risk include the unknown rates of asymptomatic maternal infection and asymptomatic fetal infection, as well as the limitations in accessing the conceptus for definitive diagnosis without invasive testing (such as amniocentesis) and the attendant risks, including pregnancy loss.

Through mathematical modelling using data from the French Polynesia outbreak of all potentially infected pregnant women, both symptomatic and asymptomatic, the estimated risk of microcephaly (defined as head circumference [HC] less than $-2 \mathrm{SD}$ ) with maternal ZIKV infection was $1 \%$, with the highest risk occurring in first-trimester infections. ${ }^{(19)}$ Other fetal abnormalities, including non-microcephalic CNS anomalies, that potentially constitute CZS were not included in this estimate. This concurs with the most recent modelling based on ZIKV infection in Bahia (reporting the highest rates of $\mathrm{CZS}$ ), in the Northeast Region of Brazil, which predicted the risks of microcephaly to be $0.88 \%$ to $13.2 \%$, depending on an assumed range of infection rate of $80 \%$ down to $10 \%{ }^{(48)}$ Another modelling study using epidemiological data from Brazil estimated the risk of microcephaly ( $\mathrm{HC}$ less than $-2 \mathrm{SD}$ ) to be $46.7 \%$ (confidence interval $14 \%-100 \%$ ) following only symptomatic first-trimester ZIKV infection, ${ }^{(49)}$ thus suggesting that symptoms may be an indicator of a poor prognosis.

In a separate prospective Brazilian cohort comprising only symptomatic ZIKV-confirmed pregnant cases $(n=72)$, the risk of developing any of the CZS features was $29 \%$ when infection occurred from 6-35 weeks of gestation, including $17 \%$ of cases demonstrating intracranial and ophthalmic anomalies, and $21 \%$ displaying features suggestive of fetal growth restriction, placental malfunction or fetal death. ${ }^{(19)}$ This is in contrast to the 1,850 pregnant women with symptomatic ZIKV infection in Columbia:(21) 532 were diagnosed in the first trimester, 702 in the second trimester and 616 in the third trimester. Of the cases infected in the third trimester, $93 \%$ of women had delivered and none of the infants had microcephaly or brain anomalies. There was also no significant excess risk of low birth weight $(2 \%)$, preterm delivery $(8 \%)$ or perinatal demise $(1 \%) .{ }^{(21)}$

It remains to be seen if the presence of maternal symptoms is truly an adverse prognostic sign for pregnancy, as has been suggested for maternal dengue infection. ${ }^{(50)}$ Retrospective Brazilian case series of infants with features of presumed CZS suggest a high symptomatic rate in $70 \%-80 \%$ of mothers during pregnancy. ${ }^{(28,29,31)}$ This should be cautiously interpreted, 


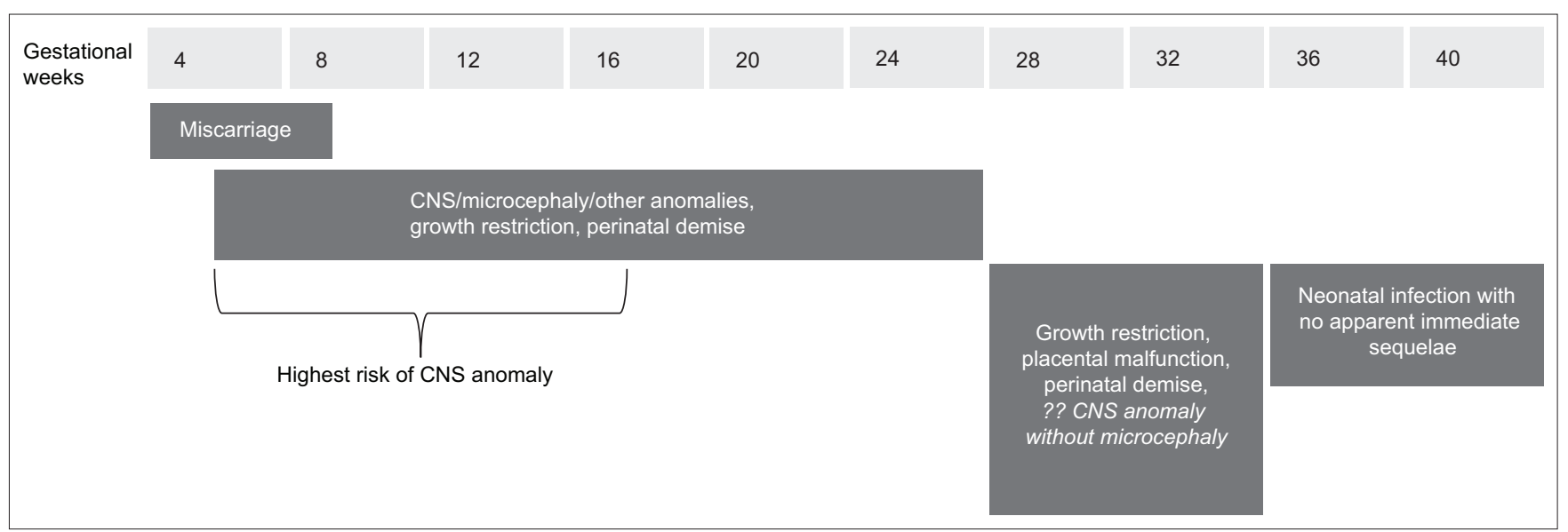

Fig. 1 Chart shows the gestational timing of maternal infection and fetal phenotype. Based on current knowledge, ${ }^{(18,21,22,64)}$ if vertical transmission of Zika infection occurs, the possible risks to the pregnancy and fetus associated with a particular gestational timing of the maternal infection are summarised. The absolute risks to the fetus during each of these window periods remain to be determined, but overall, they are believed to be relatively low, with the highest risk in the first ${ }^{(19)}$ and early second trimesters. ${ }^{\left({ }^{3}\right)}$ The light grey boxes indicate the timing of maternal infection (in gestational weeks), while the dark grey boxes show the resultant anomalies for each time period. The text in italics represents retrospective information on the presumed timing of the maternal infection, which is less accurate. ${ }^{(48)}$ CNS: central nervous system.

as ascertainment bias is inherent in such data, which relies on subject recall following an adverse event and thus tends to overestimate symptomatology rates. Conversely, a recent report from Columbia found that, so far, all four microcephaly cases (HC less than $-3 \mathrm{SD}$ ) that tested positive for ZIKV in the neonate occurred in mothers who were asymptomatic during pregnancy. ${ }^{(21)}$ At the time of the current study, data from Columbia for those infected in the first trimester is still forthcoming.

At the individual level, the estimated risk of vertical transmission in a woman infected with ZIKV is, at most, comparable to that for other well-studied viral infections, although the risk may turn out to be much lower. With maternal cytomegalovirus (CMV) infections, $30 \%-40 \%$ of fetuses become infected; of these, only about $12 \%$ develop signs and symptoms of congenital CMV syndrome, ${ }^{(51)}$ while the risk of congenital rubella syndrome with first-trimester maternal infection could be greater than $50 \% .{ }^{(52)}$ From a population-level perspective, however, it is helpful to note that the absolute risk of a baby being born with microcephaly in an area with the highest incidence of ZIKV infections in Brazil is estimated to be only four in 1,000. ${ }^{(51)}$

\section{GESTATIONAL TIMING OF ZIKV INFECTION AND FEATURES OF CZS}

Vertical transmission of ZIKV has been documented at all stages of pregnancy, and there are emerging patterns in the manifestation of the CZS phenotype that correlate to the gestational timing of maternal ZIKV infection (Fig. 1). ${ }^{(18,22)}$ The highest risk period for CNS damage has been proposed to be maternal infection in the first trimester, ${ }^{(19,48)}$ or $14-17$ weeks, ${ }^{(53)}$ while the impact on fetal growth and well-being may continue to occur with maternal infection well into the third trimester. ${ }^{(18)}$ In the largest study of affected neonates, among the 183 cases of definite or probable CZS with microcephaly, $77 \%$ of the pregnant women retrospectively reported having a rash in the first trimester, $18 \%$ in the second trimester and 5\% in the third trimester, ${ }^{(22)}$ in agreement with mathematical models that the greatest risk occurs in first- trimester infection. Women infected before 7-8 weeks of gestation are at risk of miscarriage, ${ }^{(18,27,54)}$ with a few cases being confirmed by the finding of positive ZIKV RT-PCR in the maternal serum and products of conception. ${ }^{(54)}$ However, in the Columbian study, the pregnancies of $84 \%$ of the 532 women who were infected in the first trimester were still ongoing(21) at the time of publication; this is inconsistent with an excessive miscarriage risk as compared with the general Western population. ${ }^{(55)}$ It is possible that many who had miscarried were not included in the cohort, thus resulting in an underestimation of miscarriage risk.

Cases of congenital ZIKV infection with ultrasonographic evidence of microcephaly also demonstrated a high prevalence of CNS malformation. ${ }^{(56)}$ Although it has been proposed that CNS anomalies without microcephaly may develop with maternal infection after 30 weeks of gestation, ${ }^{(22)}$ the strength of evidence for this is weak, since the possible timing of infection was retrospectively ascertained. In prospective studies, CNS anomalies were limited to maternal infections occurring at 8-27 weeks of gestation, with no reported CNS involvement for infections beyond this period. ${ }^{(18,21,57-59)}$ Such a gestational window coincides with the period of radial neuromigration of neural progenitor cells from the periventricular germinal matrix to the cortical plate, which is estimated to end at around 28-30 weeks of gestation. ${ }^{(60)}$ High expression of the AXL receptor, which mediates cellular entry of ZIKV, has been reported in human fetal radial glial cells, ${ }^{(61)}$ suggesting that these cells are the primary targets that are most vulnerable to ZIKV entry. ${ }^{(57)}$ Impairment in the function of these cells could underlie the typical CZS manifestations of reduced cortical volume, ventriculomegaly and hypogyration. This hypothesis is consistent with histopathological findings, ${ }^{(17)}$ neonatal neuroimaging findings, ${ }^{(58,59)}$ in vitro results from ZIKV-infected human brain organoids generated from induced pluripotent stem cells, and in vivo maternal infection in a mouse model. ${ }^{(44)}$ The neurotropic characteristic of ZIKV is evident, with the highest viral loads reported in brain tissue on human fetal postmortems, with little or none in the heart, lung and liver. ${ }^{(17)}$ The 
retina also demonstrates high $\mathrm{AXL}$ expression, ${ }^{(58)}$ and ophthalmic anomalies of gross pigment mottling, chorioretinal atrophy and optic nerve hypoplasia have been documented in CZS. ${ }^{(29,30,61)}$

On the other hand, placental-related manifestations or growth problems related to congenital ZIKV infection could happen in about $20 \%$ of cases of maternal ZIKV infection occurring at any time during the pregnancy (up to 35 weeks), ${ }^{(4,18)}$ even in the absence of brain anomalies. The presence of a high placental viral load in some cases ${ }^{(54)}$ also supports the causal link between ZIKV infection and placental malfunction. There have been suggestions that ZIKV can be harboured within the macrophages of placental tissue, ${ }^{(62)}$ with multifocal calcifications ${ }^{(17)}$ and reduced placental-fetal weight ratios, ${ }^{(17)}$ which could lead to placental insufficiency. Reported ultrasonographic and clinical findings include intrauterine growth restriction, oligohydramnios, abnormal Doppler waveforms of the umbilical artery or middle cerebral artery, and widespread placental calcification. ${ }^{(17,18)}$ Perinatal losses, including miscarriages, late intrauterine demise and early neonatal deaths, have also been documented. ${ }^{(18,62,63)}$ Few cases of maternal ZIKV infection occurring at term with selflimiting symptoms in the neonate have been reported ${ }^{(64)}$ but no associated CZS cases have been documented to date. The overall perinatal mortality rate in Brazil has not increased significantly since the ZIKV outbreak, and when considered together with the Columbian data, there is little evidence to suggest that, even at the height of an outbreak, ZIKV infection at term is a significant contributor to perinatal demise.

Although ZIKV has been documented in the breast milk of acutely infected women, ${ }^{(65)}$ no clinically impactful vertical transmission from breastfeeding has been documented. The $\mathrm{ACOG}^{(66)}$ has advised that the benefits of breastfeeding outweigh any possible risk.

\section{CHALLENGES OF DIAGNOSING AND MANAGING CZS ANTENATALLY}

In addition to routine ultrasonography (USS) to detect fetal anomalies in the mid-trimester, the Singapore $\mathrm{MOH}$ has also recommended further serial USS for monitoring of fetal growth, CNS and other anomalies in pregnant women with confirmed ZIKV infection. In areas of active local ZIKV transmission or following possible ZIKV exposure, if a symptomatic pregnant woman remains anxious despite negative RT-PCR results, more frequent fetal surveillance can be offered. ACOG has gone further to suggest that, regardless of symptoms or ZIKV testing results, all exposed women should be offered serial USS at four-weekly intervals throughout their pregnancy. ${ }^{(66)}$

Theoretically, following maternal ZIKV infection, time is required to breach the placental barrier and for fetal ZIKV infection to result in structural damage that can be detectable by USS. Following maternal ZIKV infection in the first trimester, the earliest USS detection of a CNS anomaly was reported at about 19 weeks, ${ }^{(25)}$ and the earliest detection of intrauterine growth restriction was at around 18 weeks. ${ }^{(63)}$ However, following a second-trimester infection, the shortest interval reported between maternal infection and detectable CNS anomalies was three weeks (infection at 18 weeks and USS manifestation at 21 weeks). ${ }^{(41)}$ CZS may take longer than two months to be detectable by USS, ${ }^{(17)}$ but the limitations of existing reports lie in the lack of serial USS; abnormalities were only detected when the woman was able to access an imaging facility. Hence, following early gestational maternal ZIKV infection, serial USS surveillance for CZS should begin at 18-20 weeks. ${ }^{(66)}$ CNS anomalies detectable by USS include significantly reduced growth velocity of the head circumference, microcephaly, ventriculomegaly, small/absent cavum septum pellucidum, asymmetrical cerebral hemispheres, hypoplastic cerebellum, partial/complete agenesis of the cerebellar vermis, microcalcifications and prominent choroid plexus. ${ }^{(16)}$ Postnatally, these structural anomalies have also been confirmed by computed tomography and magnetic resonance imaging in affected neonates, ${ }^{(59)}$ and postmortem for those who had succumbed. ${ }^{(57)}$ In addition, neurodevelopmental testing of affected neonates has revealed abnormal hearing and vision, decreased muscle tone, spasticity, psychomotor delay, brainstem dysfunction with sucking or swallowing difficulties, seizures, arthrogryposis, and amyoplasia of the lower limbs. ${ }^{(21)}$ Even if the neonate is phenotypically normal at birth, full knowledge of the long-term outcome of those exposed to in utero ZIKV must await the follow-up of current birth cohorts.

Pregnancies with confirmed maternal ZIKV infection and cases with USS evidence suggestive of CZS should be referred to maternal-fetal medicine specialists. ${ }^{(37)}$ Antenatally, potential or suspected CZS cases may be offered amniocentesis to look for the presence of ZIKV in the amniotic fluid; however, the sensitivity and specificity of this test is unknown, and even if the result is positive, the extent of fetal functional impairment and long-term prognosis in the absence of fetal anomalies is difficult to predict with current knowledge. Similarly, it is not known how soon the amniotic fluid is expected to become RT-PCR positive for ZIKV after maternal infection and the length of time RT-PCR remains positive for ZIKV after fetal infection. The shortest reported interval between maternal ZIKV infection and a positive RT-PCR result on amniotic fluid testing was four weeks in a case where no fetal anomalies were detected by USS. ${ }^{(39)}$ Therefore, the Singapore $\mathrm{MOH}$, ACOG and CDC advise that amniocentesis should only be performed after proper counselling and offered on a case-bycase basis, as with other suspected viral infections in pregnancy.

There is currently no available vaccine or treatment to prevent or treat CZS. Unfortunately, the damaging pathophysiological effects of ZIKV infection precede the clinical detection of CZS. Highly restrictive termination of pregnancy laws are common in countries where Aedes mosquitoes breed. South America has already seen a sharp increase in Internet orders for 'abortion pills' in the wake of the ZIKV outbreak. In countries like Singapore, the upper limit for termination of pregnancy is set at 24 weeks of gestation. This could pose a dilemma for parents who have to make decisions regarding termination before USS detection of fetal anomalies, which may only emerge beyond this gestational period. The psychosocial morbidity arising from terminations, especially in the face of such uncertainty, cannot be underestimated. ${ }^{(67)}$ 
In view of this, systematic gathering of data is the key to improving our approach to future management of ZIKV infections. Furthermore, in all antenatally confirmed maternal ZIKV cases and cases in which the fetus or neonate displays features of CZS, it is important to properly document the evidence of ZIKV infection through histopathological examination and ZIKV RT-PCR of the placenta, umbilical cord and neonatal blood following delivery or termination of pregnancy, as recommended by the $\mathrm{MOH} .{ }^{(37)}$

\section{VECTOR CONTROL AND FAMILY PLANNING}

Public health measures, such as vector control, remain the mainstay of risk reduction for ZIKV infection. Whenever possible, pregnant women should avoid visiting areas with active ZIKV transmission, especially in the first and second trimesters of pregnancy. If this is unavoidable (such as in the case of a small country like Singapore, or if the woman lives or works in such areas), she should take strict precautions to minimise mosquito bites and practise safe sex. Mosquito bites can be reduced by covering up with clothing, using insect repellents, and using nets or shutting windows to keep mosquitoes out when indoors. Vigilance in diagnosing the initial cases of ZIKV infections is critical for prompt mobilisation of vector control resources to contain the local spread of the virus. Whether additional precautions over and above those of vector control will be successful in minimising ZIKV infection in pregnant women, and hence the incidence of CZS, remains to be evaluated. These precautions include active surveillance of all individuals entering the country or returning from countries with an ongoing ZIKV outbreak or living in areas of active transmission, and isolation of viraemic individuals in air-conditioned rooms in dedicated healthcare settings to prevent transmission, as previously advocated by the Singapore $\mathrm{MOH}$ prior to and at the start of the recent outbreak. However, as $80 \%$ of ZIKV infections are thought to be asymptomatic, many infectious individuals remain undiagnosed and are therefore not confined, making such strategies ineffective.

Although sexual transmission of ZIKV has been documented and the virus has been found to be highly concentrated in semen for a protracted period, it is considered a minor route of transmission. ${ }^{(68)}$ The incubation period following sexual transmission from a man to his female partner has been reported to be $9-14$ days. ${ }^{(69,70)}$ The Singapore $\mathrm{MOH}$ has recommended that men with confirmed ZIKV infection should practise safe sex, primarily the consistent and correct use of condoms, or consider abstinence, for six months. If a man who has been potentially exposed to ZIKV has a pregnant partner, condoms should be used for the duration of the pregnancy. For men who have visited but do not normally live in areas of active local transmission, there are variations in the time frame for condom use (range 4-8 weeks), as recommended by the WHO, Public Health England, Australia's Department of Health and CDC. For confirmed ZIKV-infected women planning for pregnancy, the $\mathrm{MOH}, \mathrm{WHO}$ and CDC recommend a waiting period of eight weeks before attempting to conceive, due to evidence of viral propagation in vaginal epithelium in animal models. ${ }^{(71)}$
The $\mathrm{MOH}$ has advised that couples visiting or living in areas of active ZIKV transmission should take strict precautions against mosquito bites, but may continue planning for a pregnancy. ${ }^{(72)}$ CDC and ACOG have suggested that women in such situations should consider family planning carefully, and if a decision is made not to conceive, they should ensure that reliable contraception is used to avoid unintended pregnancies. However, if ZIKV becomes endemic in Singapore, it may be inappropriate to advise women to put pregnancy plans on hold for an indefinite length of time, in anticipation of an event that may arise sometime in the future. Furthermore, an outbreak did not ensue in the dengue-endemic country of Thailand despite a few reported cases of vector-borne ZIKV transmission. Although Thailand may already have some degree of herd immunity, this example shows that it remains unclear how long advice to avoid pregnancy, if given, should be adhered to. If or when an outbreak does occur in a Zika-naïve population, a protracted period of time might be needed for the population to attain adequate levels of herd immunity and bring the outbreak under control; this is a factor to be taken into consideration in family planning discussions. For example, in a small population of just over a quarter of a million people in French Polynesia, the outbreak of 2013-2014 lasted for almost a year, ${ }^{(11)}$ but in the large urbanised population of Brazil, the outbreak is expected to continue for much longer.

\section{CONCLUSION}

Potential exacerbating factors for a global ZIKV outbreak include the current El Niño weather condition, which has increased Aedes mosquito propagation, ${ }^{(9)}$ and in Singapore, increased population density and construction work. Hopes that an effective vaccine could be introduced before a truly global outbreak transpires remain guarded, and the prospects of a further ZIKV outbreak ${ }^{(3)}$ and increased incidence of CZS across the dengue-endemic world remain a reality. However, mathematical predictions of the risk of ZIKV infection to visitors to Brazil were extremely low in 2016, ${ }^{(73)}$ and the ZIKV outbreak in Brazil did not have any apparent impact on Singaporean athletes and supporters who returned from the Olympic Games. Reassuringly, as the absolute risk of CZS is relatively low even with an ongoing local ZIKV outbreak, clinicians should maintain a realistic perspective of the impact of ZIKV on pregnancy. Despite the uncertainties, expectant mothers can be appropriately and systematically counselled and obstetric surveillance better planned, using a framework that considers the gestational timing of maternal ZIKV infection and our current understanding of gestational windows of risk for different elements of the CZS phenotype. As more research evidence emerges, this framework can be further refined and incorporated into current algorithms of clinical care for improved management.

\section{DISCLOSURE}

Chan SY is a member of the Epigen Academic Consortium, which has received grants and non-financial support from industry funding (Abbott Nutrition, Nestle and Danone) outside of this submitted work. Tambyah PA has received grants from Sanofi Pasteur, GlaxoSmithKline and Fab'entech outside of this submitted work. 


\section{REFERENCES}

1. World Health Organization. Zika situation report. Available at: http:// www.who.int/emergencies/zika-virus/situation-report/5-february-2016/ en/. Accessed February 5, 2016

2. Dick GW, Kitchen SF, Haddow AJ. Zika virus. I. Isolations and serological specificity. Trans R Soc Trop Med Hyg 1952; 46:509-20.

3. Dick GW. Zika virus. II. Pathogenicity and physical properties. Trans R Soc Trop Med Hyg 1952; 46:521-34.

4. Buathong R, Hermann L, Thaisomboonsuk B, et al. Detection of Zika virus infection in Thailand, 2015. Am J Trop Med Hyg 2015; 93:380-3.

5. Marchette NJ, Garcia R, Rudnick A. Isolation of Zika virus from Aedes aegypti mosquitoes in Malaysia. Am J Trop Med Hyg 1969; 18:411-5.

6. Olson JG, Ksiazek TG, Suhandiman, Triwibowo. Zika virus, a cause of fever in Central Java, Indonesia. Trans R Soc Trop Med Hyg. 1981; 75:389-93.

7. Meltzer E, Lustig Y, Leshem E, et al. Zika virus disease in traveler returning from Vietnam to Israel. Emerg Infect Dis 2016; 22:1521-2.

8. Mo Y, Alferez Salada BM, Tambyah PA. Zika virus-a review for clinicians Br Med Bull 2016; 119:25-36.

9. Heft DE, Walton WE. Effects of the El Nino--Southern Oscillation (ENSO) cycle on mosquito populations in southern California. J Vector Ecol 2008; 33:17-29.

10. Duffy MR, Chen TH, Hancock WT, et al. Zika virus outbreak on Yap Island, Federated States of Micronesia. N Engl J Med 2009; 360:2536-43.

11. Cao-Lormeau VM, Roche C, Teissier A, et al. Zika virus, French polynesia, South pacific, 2013. Emerg Infect Dis 2014; 20:1085-6.

12. Musso D, Cao-Lormeau VM, Gubler DJ. Zika virus: following the path of dengue and chikungunya? Lancet 2015; 386:243-4.

13. Musso D. Zika virus transmission from French Polynesia to Brazil. Emerg Infect Dis 2015; 21:1887.

14. Campos GS, Bandeira AC, Sardi SI. Zika virus outbreak, Bahia, Brazil. Emerg Infect Dis 2015; 21:1885-6.

15. World Health Organisation. Zika situation report. Available at: http:// www.who.int/emergencies/zika-virus/situation-report/19-may-2016/en/. Accessed May 19, 2016.

16. Oliveira Melo AS, Malinger G, Ximenes R, et al. Zika virus intrauterine infection causes fetal brain abnormality and microcephaly: tip of the iceberg? Ultrasound Obstet Gynecol 2016; 47:6-7.

17. Mlakar J, Korva M, Tul N, et al. Zika Virus associated with microcephaly. N Engl J Med 2016; 374:951-8.

18. Brasil P, Pereira JP Jr., Raja Gabaglia C, et al. Zika virus infection in pregnant women in Rio de Janeiro - Preliminary Report. N Engl J Med 2016.

19. Cauchemez S, Besnard M, Bompard P, et al. Association between Zika virus and microcephaly in French Polynesia, 2013-15: a retrospective study. Lancet 2016; 387:2125-32.

20. Ministry of Health, Brazil. [Microcephaly - Ministry of Health releases epidemiological bulletin]. Available at: http://portalsaude.saude.gov. br/index.php/cidadao/principal/agencia-saude/20805-ministerio-dasaude-divulga-boletim-epidemiologico. Accessed December 17, 2015. Portuguese.

21. Pacheco O, Beltran $M$, Nelson CA, et al. Zika virus disease in Colombia - Preliminary Report. N Engl J Med 2016.

22. Franca GV, Schuler-Faccini L, Oliveira WK, et al. Congenital Zika virus syndrome in Brazil: a case series of the first 1501 livebirths with complete investigation. Lancet 2016; 388:891-7.

23. European Centre for Disease Prevention and Control: Factsheet for health professional. Updated June 23, 2016. Available at: http://ecdc.europa.eu/ en/healthtopics/zika_virus_infection/factsheet-health-professionals/pages/ factsheet_health_professionals.aspx. Accessed August 14, 2016.

24. Lessler J, Chaisson LH, Kucirka LM, et al. Assessing the global threat from Zika virus. Science 2016; 353:aaf8160.

25. Driggers RW, Ho CY, Korhonen EM, et al. Zika virus infection with prolonged maternal viremia and fetal brain abnormalities. N Engl J Med 2016; 374:2142-51

26. Mallet HP, Vial AL, Musso D. [Bilan de l'epidemie a virus Zika en polynesie francaise. 2013-2014. Bises Bulletin D'informaion Sanitaires Epidemiologiques Et Statistiques 2015]. French. Available at: http://www hygiene-publique.gov.pf/IMG/pdf/no13_-_mai_2015_-_zika.pdf. Accessed August 14, 2016.

27. Zlka virus infection: global update on epidemiology and potentially associated clinical manifestation. Wkly Epidemiol Rec 2016; 91:73-81.

28. Villamil-Gomez WE, Mendoza-Guete A, Villalobos E, et al. Diagnosis, management and follow-up of pregnant women with Zika virus infection: a preliminary report of the ZIKERNCOL cohort study on Sincelejo, Colombia. Travel Med Infect Dis 2016; 14:155-8.
29. Ventura CV, Maia M, Dias N, Ventura LO, Belfort R Jr. Zika: neurological and ocular findings in infant without microcephaly. Lancet 2016; 387:2502.

30. Petersen LR, Jamieson DJ, Powers AM, Honein MA. Zika virus. N Engl J Med 2016; 374:1552-63.

31. de Paula Freitas B, de Oliveira Dias JR, Prazeres J, et al. Ocular findings in infants with microcephaly associated with presumed Zika virus congenital infection in Salvador, Brazil. JAMA Ophthalmol 2016 Feb 9.

32. Brasil P, Sequeira PC, Freitas AD, et al. Guillain-Barre syndrome associated with Zika virus infection. Lancet 2016; 387:1482

33. Paploski IA, Prates AP, Cardoso CW, et al. Time lags between exanthematous illness attributed to Zika virus, Guillain-Barres, and microcephaly, Salvador, Brazil. Emerg Infect Dis 2016; 22:1438-44.

34. Reyna-Villasmil E, Lopez-Sanchez G, Santos-Bolivar J. Guillain-Barre syndrome due to Zika virus during pregnancy. Med Clin (Barc) 2016; 146:331-2.

35. Lanciotti RS, Kosoy OL, Laven JJ, et al. Genetic and serologic properties of Zika virus associated with an epidemic, Yap State, Micronesia, 2007. Emerg Infect Dis 2008; 14:1232-9.

36. Centers for Disease Control and Prevention. Diagnostic Tests for Zika Virus. Available at: http://www.cdc.gov/zika/hc-providers/types-of-tests. html. Accessed August 9, 2016.

37. Ministry of Health, Singapore. $\mathrm{MOH}$ and clinical advisory group on zika and pregnancy provide guidelines for pregnant women. Available at: https://www.moh.gov.sg/content/moh_web/home/pressRoom/ pressRoomltemRelease/2016/moh-and-clinical-advisory-group-on-zikaand-pregnancy-provide-gu.html. Accessed August 30, 2016.

38. Gourinat AC, O'Connor O, Calvez E, Goarant C, Dupont-Rouzeyrol M. Detection of Zika virus in urine. Emerg Infect Dis 2015; 21:84-6.

39. Meaney-Delman D, Oduyebo T, Polen KN, et al. Prolonged detection of Zika virus RNA in pregnant women. Obstet Gynecol 2016; 128:724-30.

40. Campos Rde M, Cirne-Santos C, Meira GL, et al. Prolonged detection of Zika virus RNA in urine samples during the ongoing Zika virus epidemic in Brazil. J Clin Virol 2016; 77:69-70.

41. Calvet G, Aguiar RS, Melo AS, et al. Detection and sequencing of Zika virus from amniotic fluid of fetuses with microcephaly in Brazil: a case study. Lancet Infect Dis 2016; 16:653-60.

42. Costello A, Dua T, Duran P, et al. Defining the syndrome associated with congenital Zika virus infection. Bull World Health Organ 2016; 94:406-A.

43. Rasmussen SA, Jamieson DJ, Honein MA, Petersen LR. Zika virus and birth defects--reviewing the evidence for causality. N Engl J Med 2016; 374:1981-7.

44. Cugola FR, Fernandes IR, Russo FB, et al. The Brazilian Zika virus strain causes birth defects in experimental models. Nature 2016; 534:267-71.

45. Report from physicians in the crop-sprayed town regarding dengue-Zika, microcephaly, and massive spraying with chemical poisons. Available at: http://www.reduas.com.ar/report-from-physicians-in-the-crop-sprayedtown-regarding-dengue-zika-microcephaly-and-massive-spraying-withchemical-poisons/. Accessed February 9, 2016.

46. Butler D. Brazil asks whether Zika acts alone to cause birth defects. Nature 2016; 535:475-6.

47. Nielsen KK, Bygbjerg IC. Zika virus and hyperglycaemia in pregnancy. Lancet 2016; 387:1812.

48. Johansson MA, Mier-y-Teran-Romero L, Reefhuis J, Gilboa SM, Hills SL. Zika and the risk of microcephaly. N Engl J Med 2016; 375:1-4

49. Nishiura H, Mizumoto K, Rock KS, et al. A theoretical estimate of the risk of microcephaly during pregnancy with Zika virus infection. Epidemics 2016; 15:66-70.

50. Paixao ES, Teixeira MG, Costa Mda C, Rodrigues LC. Dengue during pregnancy and adverse fetal outcomes: a systematic review and metaanalysis. Lancet Infect Dis 2016; 16:857-65.

51. Lissauer D, Smit E, Kilby MD. Zika virus and pregnancy. BJOG 2016; 123:1258-63.

52. De Santis M, Cavaliere AF, Straface G, Caruso A. Rubella infection in pregnancy. Reprod Toxicol 2006; 21:390-8.

53. Faria NR, Azevedo Rdo S, Kraemer MU, et al. Zika virus in the Americas: early epidemiological and genetic findings. Science 2016; 352:345-9.

54. Martines RB, Bhatnagar J, Keating MK, et al. Notes from the field: evidence of Zika virus infection in brain and placental tissues from two congenitally infected newborns and two fetal losses--Brazil, 2015. MMWR Morb Mortal Wkly Rep 2016; 65:159-60.

55. Rai R, Regan L. Recurrent miscarriage. Lancet 2006; 368:601-11.

56. Carvalho FH, Cordeiro KM, Peixoto AB, et al. Associated ultrasonographic findings in fetuses with microcephaly because of suspected Zika virus (ZIKV) infection during pregnancy. Prenat Diagn 2016; 36:882-7. 
57. Guillemette-Artur P, Besnard M, Eyrolle-Guignot D, Jouannic JM, Garel C. Prenatal brain MRI of fetuses with Zika virus infection. Pediatr Radiol 2016; 46:1032-9.

58. Cavalheiro S, Lopez A, Serra S, et al. Microcephaly and Zika virus: neonatal neuroradiological aspects. Childs Nerv Syst 2016; 32:1057-60.

59. de Fatima Vasco Aragao M, van der Linden V, Brainer-Lima AM, et al. Clinical features and neuroimaging (CT and MRI) findings in presumed Zika virus related congenital infection and microcephaly: retrospective case series study. BMJ 2016; 353:i1901.

60. Gupta RK, Hasan KM, Trivedi R, et al. Diffusion tensor imaging of the developing human cerebrum. J Neurosci Res 2005; 81:172-8.

61. Nowakowski TJ, Pollen AA, Di Lullo E, Sandoval-Espinosa C, Bershteyn M, Kriegstein AR. Expression Analysis Highlights AXL as a Candidate Zika Virus Entry Receptor in Neural Stem Cells. Cell Stem Cell 2016; 18:591-6.

62. Pan American Health Organization; World Health Organisation Epidemiological Update: neurological syndrome, congenital anomalies and Zika virus infection. Available at: http://reliefweb.int/sites/reliefweb. int/files/resources/2016-jan-17-cha-epi-update-zika-virus.pdf. Accessed January 17, 2016

63. Sarno M, Sacramento GA, Khouri R, et al. Zika virus infection and stillbirths: a case of hydrops fetalis, hydranencephaly and fetal demise. PLoS Neg Trop Dis 2016; 10:e004517.

64. Besnard M, Lastere S, Teissier A, Cao-Lormeau V, Musso D. Evidence of perinatal transmission of Zika virus, French Polynesia, December 2013 and February 2014. Euro Surveill 2014; 19.

65. Dupont-Rouzeyrol M, Biron A, O'Connor O, Huguon E, Descloux E.
Infectious Zika viral particles in breastmilk. Lancet 2016; 387:1051.

66. The American Congress of Obstetricians and Gynecologists. Practice advisory on Zika virus. Available at: https://www.acog.org/About-ACOG/ News-Room/Practice-Advisories/Practice-Advisory-Interim-Guidancefor-Care-of-Obstetric-Patients-During-a-Zika-Virus-Outbreak. Accessed August 14, 2016.

67. Korenromp MJ, Christiaens GC, van den Bout J, et al. Long-term psychological consequences of pregnancy termination for fetal abnormality: a cross-sectional study. Prenat Diagn 2005; 25:253-60.

68. Musso D, Roche C, Robin E, et al. Potential sexual transmission of Zika virus. Emerg Infect Dis 2015; 21:359-61.

69. Foy BD, Kobylinski KC, Chilson Foy JL, et al. Probable non-vector-borne transmission of Zika virus, Colorado, USA. Emerg Infect Dis 2011; 17:880-2.

70. Hills SL, Russell K, Hennessey M, et al. Transmission of Zika virus through sexual contact with travelers to areas of ongoing transmission - continental United States, 2016. MMWR Morb Mortal Wkly Rep 2016; 65:215-6.

71. Yockey LJ, Varela L, Rakib T, et al. Vaginal exposure to Zika virus during pregnancy leads to fetal brain infection. Cell 2016; 166:1247-56 e4.

72. Ministry of Health, Singapore. Precautionary Measures Against Zika Virus Infection. In: National Environment Agency Press Release. Available at: https://www.moh.gov.sg/content/moh_web/home/pressRoom/ pressRoomltemRelease/2016/precautionary-measures-against-zika-virusinfection-.html. Accessed January 27, 2016.

73. Burattini MN, Coutinho FA, Lopez LF, et al. Potential exposure to Zika virus for foreign tourists during the 2016 Carnival and Olympic Games in Rio de Janeiro, Brazil. Epidemiol Infect 2016; 144:1904-6. 